\title{
System of variational inequalities with interconnected obstacles
}

\author{
R. Barkhudaryan ${ }^{\mathrm{a}}$, D.A. Gomes ${ }^{\mathrm{c}}$, H. Shahgholian ${ }^{\mathrm{d}}$ and M. Salehi ${ }^{\mathrm{e}}$

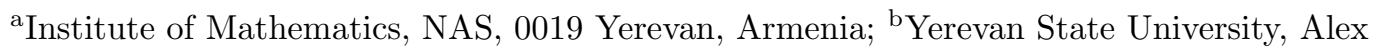 \\ Manoogian 1, 0025 Yerevan, Armenia; ${ }^{c}$ King Abdullah University of Science and Technology \\ (KAUST), CSMSE Division, Thuwal 23955-6900, Saudi Arabia; d Department of \\ Mathematics, The Royal Institute of Technology 10044 Stockholm ${ }^{\text {eDepartment of }}$ \\ Mathematics, Statistics and Physics, Qatar University, P.O. Box 2713, Doha, Qatar;
}

\section{ARTICLE HISTORY}

Compiled October 16, 2019

\begin{abstract}
Our objective with this paper is to discuss multi-switching problems, arising as variational inequalities, that models decision under uncertainty. We prove general existence theory through monotone scheme, and discuss iterative methods for numerical results. We also connect the recently developed models for asset bubbles (which is a non-local problem) to switching problems with two possible switching cases.
\end{abstract}

\section{KEYWORDS}

Optimal switching; free boundaries; viscosity solution; interconnected obstacles

AMS CLASSIFICATION

60G40; 49L25; 35R35; 93E20

\section{Introduction}

\subsection{Background}

In this paper, we examine interconnected obstacle problems and develop a numerical approximation scheme. These problems are given as (weakly coupled) systems of variational inequalities and model optimal decision or switching under uncertainty. For example, these problems can be regarded as a system-version of the Americantype option problem with multiple choices and switching between them. The following problems are typical applications where the need for optimal switching and decision making arises:

- deciding whether to use gas, oil, or carbon fuel for heating system of a city;

- concluding whether to keep a mine open or closed, depending on the market price of a commodity;

- evaluating asset price bubbles arising from overconfidence that, in turn, is caused by a difference in beliefs between groups of traders. 
The above problems are well studied from a stochastic point of view and lead to the systems of variational inequalities with interconnected obstacles. Those systems can be written (for vector-valued functions $\mathbf{u}=\left(u_{1}, \cdots, u_{m}\right)$ ) as

$$
\min \left(-L^{i} u_{i}-f_{i}, \min _{j \neq i}\left(u_{i}-u_{j}+\psi_{i j}\right)\right)=0,\left.\quad u_{i}\right|_{\partial \Omega}=g_{i}
$$

for $i=1, \ldots, m$ and with the compatibility condition on $\partial \Omega, g_{i}-g_{j}+\psi_{i j} \geq 0, i \neq j \geq 0$. One has also to assume certain no-gain on the loop condition (see (5)). A solution to the preceding system is understood in the viscosity sense, as we discuss later.

Here, we examine the above mentioned optimal switching problem from different perspectives focusing primarily on an iterative method and a monotone scheme. We begin by addressing the theory of viscosity solutions for these systems and show the equivalence between several definitions. Next, we introduce a monotone scheme that we use to study the existence of solutions and for numerical computations. Subsequently, we examine an application in the theory of financial bubbles. Finally, we discuss several concrete examples and corresponding numerical simulations.

\subsection{Historical development}

Systems of fully nonlinear weakly coupled concave elliptic and parabolic equations have been investigated extensively. The Dirichlet problem for the Bellman equation and its connection with optimal switching problems was studied in [1]. Further results within the framework of viscosity solutions, namely existence, uniqueness and $W^{2, \infty}$ regularity, were established in [2-5]. Subsequently, existence and uniqueness for viscosity solution of monotone systems of elliptic PDEs was considered in [6,7], and for weakly coupled systems under more general hypothesis in $[8,9]$. These results were further extended for parabolic problems in [10]. Bilateral obstacle problems were studied in [11]. Additional uniqueness results for first-order weakly coupled systems with interconnected obstacles were considered in [12].

These systems arise in the optimal control of hybrid systems and in controlled Markov process with random switching; see, [13,14], and references therein. The starting-stopping problem and applications to finance were considered in [15]. Finally, weakly coupled reaction-diffusion systems have also been studied in [16], in the context of wave-front propagation.

The solvability of quasi-monotone weakly coupled systems of uniformly elliptic PDEs of Isaac's type using viscosity solutions methods was examined in [17]. Many classical estimates have been extended for these systems. For example, the authors of [18] establish Harnack-type inequalities for viscosity solutions of fully nonlinear cooperative elliptic systems, and, in [19], the authors consider Liouville-type theorems and a priori estimates for solutions of boundary value problems for systems of elliptic PDEs. More results connected with optimal switching problems with signed switching costs can be found in [20-25].

\subsection{Mathematical Setting}

Let $\Omega \subset \mathbb{R}^{d}$ be a bounded smooth domain. We consider an agent that can be anywhere in $\Omega$ and in any one of a finite number, $p$, of states. For every $1 \leq i \leq p$, the agent 
moves in $\Omega$ according to a diffusion:

$$
d x=b_{i}(x) d t+\sigma_{i}(x) d W_{t},
$$

where $W_{t}$ is a Brownian motion in a suitable probability space, $b_{i}: \Omega \rightarrow \mathbb{R}^{n}$ and $\sigma_{i}: \Omega \rightarrow \mathbb{R}^{n \times m}$ are smooth functions. This agent can switch from any diffusion mode to another. At every instant, the agent pays a running cost, $-f_{i(t)}(x(t))$, that depends on the present state, $i(t)$, and position, $x(t)$. When changing from state $i$ to state $j$, the agent incurs in a transition cost, $-\psi_{i j}(x(t))$. Finally, if the diffusion reaches the boundary at $x \in \partial \Omega$ and the agent is in state $i$, the process is stopped and a cost, $-h_{i}(x)$, is incurred. The traditional variational approach to optimal switching is maximization of a profit functional, the negative of the cost. This functional is

$$
\begin{aligned}
u_{i}(x)=\max _{i(t), i(0)=i} E\left[\int_{0}^{T_{\partial \Omega}} f_{i(t)}(x(t)) d t\right. & \\
& \left.-\sum_{t \leq T_{\partial \Omega}} \psi_{i\left(t^{-}\right), i\left(t^{+}\right)}(x(t))+g_{i\left(T_{\partial \Omega}\right)}\left(x\left(T_{\partial \Omega}\right)\right)\right],
\end{aligned}
$$

where $T_{\partial \Omega}$ denotes the exit time from $\Omega$. Additionally, we use the convention that $\psi_{i i}=0$. Therefore, if the number of jumps from one state to another (jumps happen when $\left.i\left(t^{-}\right) \neq i\left(t^{+}\right)\right)$is finite or countable, the sum is well defined. We observe, however, that this sum may diverge. Finally, we assume that $x(t)$ is a (continuous) solution to (1) with $i=i(t)$; that is, when changing from mode $i$ to mode $j$, the state $x$ does not change - only its dynamics is changed.

The generator of the diffusion in (1) is

$$
L^{i} v=\frac{1}{2} \sigma_{i} \sigma_{i}^{T}: D^{2} v+b_{i} \cdot D v .
$$

As discussed in [2,3], the value function $u_{i}$ of the optimal switching problem $(2)$ is a viscosity solution (as defined in the next section) of the following system of variational inequalities:

$$
\left\{\begin{array}{l}
\min \left(-L^{i} u_{i}-f_{i}, \min _{j \neq i}\left(u_{i}-u_{j}+\psi_{i j}\right)\right)=0, \\
\left.u_{i}\right|_{\partial \Omega}=g_{i}, \quad i=1, \ldots, m
\end{array}\right.
$$

In addition, we assume the following boundary compatibility condition:

$$
g_{i}-g_{j}+\psi_{i j} \geq 0, \quad i \neq j \geq 0
$$

on $\partial \Omega$.

For the sake of simplicity, we consider the case where $L^{i} \equiv L^{j}$. Moreover, for the optimal switching problem to be well defined, we impose a non-profit loop condition; that is, for any $x \in \Omega$ and any loop $i_{0}, i_{1}, \ldots i_{p}=i_{0}$, where $2 \leq p \leq n$, we assume that

$$
\sum_{j=1}^{p} \psi_{i_{j-1}, i_{j}} \geq 0
$$


A simple argument shows that the former condition is necessary for (3) to have a solution. In the literature, this condition is also called as no loop condition (see, $[5,7]$ ). Under this condition, the agents cannot make arbitrary gains by looping. In general, the non-profit loop condition does not guarantee uniqueness. For the comparison principle to be valid and for the uniqueness of solution, we need the following stricter condition to hold:

$$
\sum_{j=1}^{p} \psi_{i_{j-1}, i_{j}}>\gamma>0 .
$$

It is easy to show the system (3) and the dynamic programming problem (2) model the same phenomenon and that any solution to one of them is also a solution to the other one, provided the solution is smooth enough. We state and prove the verification part of this well-known result as a theorem for future reference. Later, we revisit this result in the context of viscosity solutions under continuity assumptions.

Theorem 1.1 (Public domain). Any smooth enough solution of the optimal switching problem (3) is the value function in (2).

It is also well-known that any smooth enough function represented by (2) solves (3). However, in general, the value function in (2) is not smooth enough and, thus, this issue needs to be tackled with viscosity solutions.

Proof. Let $u=\left(u_{1}, u_{2}, \ldots, u_{m}\right)$ solves the system of variational inequalities (3). Take any control, $i(\cdot)$, and consider the corresponding cost function

$$
\begin{array}{r}
E\left[\int_{0}^{T_{\partial \Omega}} f_{i(t)}(x(t)) d t-\sum_{t \leq T_{\partial \Omega}} \psi_{i\left(t^{-}\right), i\left(t^{+}\right)}(x(t))+g_{i\left(T_{\partial \Omega}\right)}\left(x\left(T_{\partial \Omega}\right)\right)\right] \leq \\
-E \int_{0}^{T \partial \Omega} L^{i(t)} u_{i(t)}(x(t)) d t+E \sum_{t \leq T_{\partial \Omega}}\left[u_{i(t-)}(x(t))-u_{i(t+)}(x(t))\right] \\
+E g_{i\left(T_{\partial \Omega}\right)} x\left(T_{\partial \Omega}\right)=u_{i}(x) .
\end{array}
$$

The last equality follows from the Dynkin formula. Next, we choose the optimal control: if $L^{i} u_{i}-f_{i}=0$, then it is optimal to continue the process; otherwise, we switch from regime $i$ to $j$, where $j$ is such that $u_{i}-u_{j}+\psi_{i j}=0$. This choice of control guarantees the equality in the above inequality.

\section{Viscosity solutions}

Here, we review the basic definitions in the theory of viscosity solutions for weakly coupled systems.

We consider a system of $m$ equations of the following type

$$
F^{i}\left(D_{x x}^{2} u_{i}, D_{x} u_{i}, \Lambda^{i} \mathbf{u}, u_{i}, x\right)=0, \quad \text { in } \Omega, \quad i=1, \ldots m,
$$

where $\Omega$ is a bounded open subset of $\mathbb{R}^{d}$. For each $1 \leq i \leq m, F^{i}$ is an elliptic concave second-order partial differential operator, and the $j$-th component of the coupling 
operator $\Lambda^{i}$ is defined as $\left(\Lambda^{i} \mathbf{u}\right)_{j}=u_{i}-u_{j}$.

For each $1 \leq i \leq m$, let $F^{i}: \mathbb{R}^{d \times d} \times \mathbb{R}^{d} \times \mathbb{R}^{m} \times \mathbb{R} \times \Omega \rightarrow \mathbb{R}$, where each $F^{i}$ is continuous. Furthermore, we assume the following ellipticity condition: if $M, N$ are $n \times n$ symmetric matrices and $M \leq N$, then

$$
F^{i}(M, p, \lambda, z, x) \geq F^{i}(N, p, \lambda, z, x) .
$$

The function $F$ is uniformly elliptic if there exist constants $0<\theta \leq \Theta$ such that, for all $1 \leq i \leq m$,

$$
\theta\|N\| \leq F^{i}(M, p, \lambda, z, x)-F^{i}(M+N, p, \lambda, z, x) \leq \Theta\|N\|,
$$

whenever $N$ is a positive semi-definite symmetric matrix. If $F^{i}$ is differentiable, the preceding inequality is equivalent to

$$
\theta \leq-D_{M} F^{i}(M, p, \lambda, z, x) \leq \Theta .
$$

Concerning the dependence of $F$ in $z$, we suppose that, for each $1 \leq i \leq m$, $F^{i}(M, p, \lambda, z, x)$ is increasing in $z$ and that $D_{z} F^{i}$ is uniformly bounded. In addition, we require that for all $1 \leq i \leq m, F^{i}$ is monotone in $\lambda$; that is, that $D_{\lambda_{j}} F^{i} \geq 0$, for $j \neq i$. Finally, we assume that $D_{p} F$ is bounded and that $D_{z} F \leq 0$. We suppose further that $F$ is Lipschitz in $x$. Because we are dealing with optimal switching problems, we also require that

$$
(M, p, \lambda) \mapsto F(M, p, \lambda, z, x)
$$

is concave for each $z$ and $x$.

A first case that satisfies the above conditions is the following: given positive-definite matrices $a_{j k}^{i}(x), b_{i j} \geq 0$, and $H^{i}(x, p)$, we consider

$$
u_{i}-a_{j k}^{i} \partial_{x_{j} x_{k}} u_{i}+H^{i}\left(x, D u_{i}\right)+b_{i k}\left(u_{i}-u_{j}\right)=0 .
$$

A second example is

$$
\min \left(-a_{j k}^{i} \partial_{x_{j} x_{k}} u_{i}+H^{i}\left(x, D u_{i}\right), u_{i}-u_{j}+\psi_{i j}\right)=0
$$

where $H$ is a continuous function.

Definition 2.1 (Continuous viscosity solution). Let $\mathbf{u}: \Omega \rightarrow \mathbb{R}^{m}$ be a bounded uniformly continuous function.

- We say that $\mathbf{u}$ is a viscosity sub-solution of (7) if for any smooth function $\varphi$ : $\Omega \rightarrow \mathbb{R}^{m}$ and any

$$
\left(x_{0}, i_{0}\right) \in \underset{(x, i) \in \Omega \times\{1, \ldots, m\}}{\arg \min }\left(u_{i}(x)-\varphi_{i}(x)\right)
$$

we have

$$
F^{i_{0}}\left(D_{x x}^{2} \varphi_{i_{0}}\left(x_{0}\right), D_{x} \varphi_{i_{0}}\left(x_{0}\right), \Lambda^{i_{0}} \varphi\left(x_{0}\right), u_{i_{0}}\left(x_{0}\right), x_{0}\right) \leq 0 .
$$


- Similarly, we say that $\mathbf{u}$ is a viscosity supersolution of (7), if we have the opposite inequality; that is, for all smooth $\varphi$ and all

$$
\left(x_{0}, i_{0}\right) \in \underset{(x, i) \in \Omega \times\{1, \ldots, m\}}{\arg \max }\left(u_{i}(x)-\varphi_{i}(x)\right),
$$

we have

$$
F^{i_{0}}\left(D_{x x}^{2} \varphi_{i_{0}}\left(x_{0}\right), D_{x} \varphi_{i_{0}}\left(x_{0}\right), \Lambda^{i_{0}} \varphi\left(x_{0}\right), u_{i_{0}}\left(x_{0}\right), x_{0}\right) \geq 0
$$

- We say that $\mathbf{u}$ is a viscosity solution of (7) if $\mathbf{u}$ is both a viscosity sub- and supersolution of (7).

A smooth enough viscosity solution to (7) is a classical solution. The converse is also true; that is, a bounded $C^{2}$ classical solution of (7) is a viscosity solution. The proof of this last fact is similar to the scalar case. However, because we are dealing with systems, the definition is less standard. Thus, for completeness, we briefly sketch the proof. For that, we take a $C^{2}$ function, $\varphi: \Omega \rightarrow \mathbb{R}^{m}$. Suppose that $u_{i}-\varphi_{i}$ attains a maximum at $\left(x_{0}, i_{0}\right)$. Then, as in the scalar case

$$
D_{x} \varphi_{i_{0}}\left(x_{0}\right)=D_{x} u_{i_{0}}\left(x_{0}\right)
$$

and

$$
D_{x x}^{2} \varphi_{i_{0}}\left(x_{0}\right) \geq D_{x x}^{2} u_{i_{0}}\left(x_{0}\right)
$$

Now, we consider the operator $\Lambda^{i_{0}}$. Because $i_{0}$ is a maximizer for $u_{i}\left(x_{0}\right)-\varphi_{i}\left(x_{0}\right)$, we have $\varphi_{i_{0}}\left(x_{0}\right)-\varphi_{j}\left(x_{0}\right) \leq u_{i_{0}}\left(x_{0}\right)-u_{j}\left(x_{0}\right)$. Therefore, $\Lambda^{i_{0}} \varphi \leq \Lambda^{i_{0}} \mathbf{u}$. According to the ellipticity and monotonicity assumptions for $F^{i}$,

$$
\begin{aligned}
F^{i_{0}}\left(D_{x x}^{2} \varphi_{i_{0}}\left(x_{0}\right), D_{x} \varphi_{i_{0}}\left(x_{0}\right)\right. & \left., \Lambda^{i_{0}} \varphi\left(x_{0}\right), u^{i_{0}}\left(x_{0}\right), x_{0}\right) \\
& \leq F^{i_{0}}\left(D_{x x}^{2} u_{i_{0}}\left(x_{0}\right), D_{x} u_{i_{0}}\left(x_{0}\right), \Lambda^{i_{0}} \mathbf{u}\left(x_{0}\right), u_{i_{0}}\left(x_{0}\right), x_{0}\right)=0,
\end{aligned}
$$

When $\left(x_{0}, i_{0}\right) \in \arg \max _{(x, i) \in \Omega \times\{1, \ldots, m\}}\left(u_{i}(x)-\varphi_{i}(x)\right)$, the case of the reverse inequality is analogous.

Later, we need to use discontinuous viscosity solutions. For that, we need the following definition.

Definition 2.2. Let $\mathbf{u}: \Omega \rightarrow \mathbb{R}^{m}$ be a bounded function. The lower semicontinuous envelope $\mathbf{u}_{*}$ of $\mathbf{u}$ is

$$
\mathbf{u}_{* i}=\liminf _{y \rightarrow x, y \in \bar{\Omega}} u_{i}(y) .
$$

Similarly, the upper semicontinuous envelope $\mathbf{u}^{*}$ of $\mathbf{u}$ is defined by

$$
\mathbf{u}^{*}{ }_{i}=\limsup _{y \rightarrow x, y \in \bar{\Omega}} u_{i}(y)
$$

A system of partial differential equation of the form (7) with the boundary condi- 
tions $u_{i}=g_{i}$ in $\partial \Omega$ can be written as

$$
G^{i}\left(D^{2} u_{i}, D u_{i}, \Lambda_{i} \mathbf{u}, u_{i}, x\right)=0 \quad \text { in } \bar{\Omega},
$$

for

$$
G^{i}(M, p, z, \lambda, x)= \begin{cases}F^{i}(M, p, z, \lambda, x) & \text { if } x \in \Omega, \\ z-g_{i}(x) & \text { if } x \in \bar{\Omega} .\end{cases}
$$

We use upper and lower semicontinuous envelopes to define $G_{*}$ and $G^{*}$

$$
\left(G_{*}\right)^{i}(M, p, z, \lambda, x)= \begin{cases}F^{i}(M, p, z, \lambda, x) & \text { if } x \in \Omega \\ \min \left(F_{i}(M, p, z, \lambda, x), z-g_{i}(x)\right) & \text { if } x \in \bar{\Omega}\end{cases}
$$

and

$$
\left(G^{*}\right)^{i}(M, p, z, \lambda, x)= \begin{cases}F_{i}(M, p, z, \lambda, x) & \text { if } x \in \Omega \\ \max \left(F_{i}(M, p, z, \lambda, x), z-g_{i}(x)\right) & \text { if } x \in \bar{\Omega}\end{cases}
$$

Definition 2.3 (Discontinuous viscosity solutions). A bounded upper semicontinuous function $\mathbf{u}$ is a viscosity subsolution of (8) if for any

$$
\left(x_{0}, i_{0}\right) \in \underset{(x, i) \in \bar{\Omega} \times\{1, \ldots, m\}}{\arg \max }\left(u_{i}(x)-\varphi_{i}(x)\right)
$$

we have

$$
\left(G_{*}\right)^{i}\left(D_{x x}^{2} \varphi_{i_{0}}\left(x_{0}\right), D_{x} \varphi_{i_{0}}\left(x_{0}\right), \Lambda^{i_{0}} \varphi\left(x_{0}\right), u_{i_{0}}\left(x_{0}\right), x_{0}\right) \leq 0 .
$$

Similarly, a bounded lower semicontinuous function $\mathbf{u}$ is a viscosity supersolution of (8) if for any

$$
\left(x_{0}, i_{0}\right) \in \underset{(x, i) \in \bar{\Omega} \times\{1, \ldots, m\}}{\arg \max }\left(u_{i}(x)-\varphi_{i}(x)\right)
$$

we have

$$
\left(G^{*}\right)^{i}\left(D_{x x}^{2} \varphi_{i_{0}}\left(x_{0}\right), D_{x} \varphi_{i_{0}}\left(x_{0}\right), \Lambda^{i_{0}} \varphi\left(x_{0}\right), u_{i_{0}}\left(x_{0}\right), x_{0}\right) \geq 0 .
$$

A bounded function $\mathbf{u}$ (not necessarily upper or lower semicontinuous) is a (discontinuous) viscosity solution of (8) if both $\mathbf{u}^{*}$ is a subsolution and $\mathbf{u}_{*}$ is a supersolution of $(8)$.

\subsection{Equivalence of viscosity solutions definitions}

Now, we compare the preceding definition of viscosity solution with the standard one by Ishii and Koike (see [7]). For that, we introduce the sets $D^{2,+} u(x)$ and $D^{2,-} u(x)$ as follows: 
Definition 2.4. For a given continuous function, $u: \Omega \rightarrow \mathbb{R}$, we define $D^{2,+} u(x)$ to be the set of all $(p, X) \in \mathbb{R}^{n} \times S^{n}$ satisfying

$$
u(y) \leq u(x)+\langle p, y-x\rangle+\frac{1}{2}\langle X(y-x), y-x\rangle+o\left(|x-y|^{2}\right),
$$

as $y \rightarrow x$. Likewise, $D^{2,-} u(x)$ is the set of all $(p, X) \in \mathbb{R}^{n} \times S^{n}$ for which

$$
u(y) \leq u(x)+\langle p, y-x\rangle+\frac{1}{2}\langle X(y-x), y-x\rangle+o\left(|x-y|^{2}\right),
$$

as $y \rightarrow x$.

Next, we recall the definition of viscosity solutions by Ishii-Koike.

Definition 2.5 (Continuous viscosity solution by Ishii and Koike). A continuous function $\mathbf{u}: \Omega \rightarrow \mathbb{R}^{m}$ is a viscosity solution to

$$
G^{i}\left(D_{x x}^{2} u_{i}, D_{x} u_{i}, \mathbf{u}, x\right)=0 \text { in } \Omega, \quad i=1,2, \ldots, m
$$

if, for all $x \in \Omega$, and $(p, X) \in D^{2,+} u_{i}(x)$, we have

$$
G^{i}(X, p, \mathbf{u}, x) \leq 0 \text { in } \Omega,
$$

and the opposite inequality holds for all $(p, X) \in D^{2,-} u_{i}(x)$.

Lemma 2.6. The two definitions of viscosity solutions, Definitions 2.4 and 2.5, are equivalent.

Proof. Here, $G^{i}\left(D_{x x}^{2} u_{i}, D_{x} u_{i}, \mathbf{u}, x\right) \equiv F^{i}\left(D_{x x}^{2} u_{i}, D_{x} u_{i}, \Lambda^{i} \mathbf{u}, u_{i}, x\right)$. First, we assume that $\mathbf{u}$ is a viscosity solution in the sense of Definition 2.1 and take $x_{0} \in \Omega$ and $(p, X) \in$ $D^{2,+} u_{i}\left(x_{0}\right)$. Then, there exists $\varphi_{i} \in C^{2}(\Omega)$ such that $(p, X)=\left(D_{x} \varphi_{i}\left(x_{0}\right), D_{x x}^{2} \varphi_{i}\left(x_{0}\right)\right)$, and $u_{i}(x)-\varphi_{i}(x)$ achieves a maximum at $x_{0}$ with $u_{i}\left(x_{0}\right)=\varphi_{i}\left(x_{0}\right)$. For each $i,\left(x_{0}, i\right) \in$ $\arg \max _{(x, j) \in \Omega \times\{1, \ldots, m\}}\left(u_{j}(x)-\varphi_{j}(x)\right)$, we have

$$
\begin{aligned}
G^{i}\left(D_{x x}^{2} \varphi_{i}\left(x_{0}\right), D_{x} \varphi_{i}\left(x_{0}\right), \varphi\left(x_{0}\right), x_{0}\right) & \\
& \equiv F^{i}\left(D_{x x}^{2} \varphi_{i}\left(x_{0}\right), D_{x} \varphi_{i}\left(x_{0}\right), \Lambda^{i} \varphi\left(x_{0}\right), \varphi_{i}\left(x_{0}\right), x_{0}\right) \leq 0 .
\end{aligned}
$$

The reverse inequality holds in the case of a minimum.

Next, we prove that if a continuous function $\mathbf{u}$ is a viscosity solution in the sense of the definition given by Ishii and Koike, then it is a viscosity solution in the sense of Definition 2.1. For any smooth function, $\varphi: \Omega \rightarrow \mathbb{R}^{m}$, and any $\left(x_{0}, i_{0}\right) \in \arg \max _{(x, j) \in \Omega \times\{1, \ldots, m\}}\left(u_{j}(x)-\varphi_{j}(x)\right)$, we have that

$$
\begin{aligned}
& 0 \geq G^{i_{0}}\left(D_{x x}^{2} \varphi_{i_{0}}\left(x_{0}\right), D_{x} \varphi_{i_{0}}\left(x_{0}\right), u\left(x_{0}\right), x_{0}\right) \\
&=F^{i_{0}}\left(D_{x x}^{2} \varphi_{i_{0}}\left(x_{0}\right), D_{x} \varphi_{i_{0}}\left(x_{0}\right), \Lambda^{i_{0}} u\left(x_{0}\right), u_{i_{0}}\left(x_{0}\right), x_{0}\right) \\
& \\
& \geq F_{i_{0}}\left(D_{x x}^{2} \varphi_{i_{0}}\left(x_{0}\right), D_{x} \varphi_{i_{0}}\left(x_{0}\right), \Lambda^{i_{0}} \varphi\left(x_{0}\right), u_{i_{0}}\left(x_{0}\right), x_{0}\right)
\end{aligned}
$$

where the last inequality is a consequence of the monotonicity of $F^{i}$. The case of the minimum can be proved similarly. 
Theorem 2.7. Let $\mathbf{u}^{\varepsilon}=\left(u_{1}^{\varepsilon}, u_{2}^{\varepsilon}, \ldots, u_{m}^{\varepsilon}\right)$ be a viscosity subsolution (supersolution) of

$$
F_{\varepsilon}^{i}\left(D_{x x}^{2} u_{i}(x), D_{x} u_{i}(x), \Lambda^{i} \mathbf{u}(x), u_{i}(x), x\right)=0, \quad \text { in } \Omega,
$$

where $F_{\varepsilon}^{i}$ are continuous functions, satisfying standard ellipticity conditions. If $F_{\varepsilon}^{i}$ converges locally uniformly on its domain to $F^{i}$ as $\varepsilon$ goes to zero, and $u_{\varepsilon}$ converges uniformly on $\bar{\Omega}$ to $\mathbf{u}=\left(u_{1}, u_{2}, \ldots, u_{m}\right)$. Then, $\mathbf{u}$ is a viscosity subsolution (supersolution) of the limiting system

$$
F^{i}\left(D_{x x}^{2} u_{i}(x), D_{x} u_{i}(x), \Lambda^{i} \mathbf{u}(x), u_{i}(x), x\right)=0, \text { in } \Omega .
$$

Proof. We fix a smooth function, $\varphi: \Omega \rightarrow \mathbb{R}^{m}$, and let $\left(x_{0}, i_{0}\right)$ be the strict maximizer of the function $(x, i) \mapsto u_{i}(x)-\varphi_{i}(x)$. We can choose $x_{\varepsilon}$ such that $\left(x_{\varepsilon}, i_{0}\right)$ is a local maximizer of $u_{i}^{\varepsilon}(x)-\varphi_{i}(x)$, and $x_{\varepsilon} \rightarrow x_{0}$ as $\varepsilon \rightarrow 0$. Indeed, take a maximizer, $x_{\varepsilon}$, of $u_{i_{0}}^{\varepsilon}(x)-\varphi_{i_{0}}(x)$. Then, for $\varepsilon$ small enough, $\left(x_{\varepsilon}, i_{0}\right)$ is a local maximizer of $u_{i}^{\varepsilon}(x)-\varphi_{i}(x)$. Consequently,

$$
F_{\varepsilon}^{i_{0}}\left(D_{x x}^{2} \varphi_{i_{0}}\left(x_{\varepsilon}\right), D_{x} \varphi_{i}\left(x_{\varepsilon}\right), \Lambda^{i} \varphi\left(x_{\varepsilon}\right), u_{i}^{\varepsilon}\left(x_{\varepsilon}\right), x_{\varepsilon}\right) \leq 0 .
$$

Therefore, by considering the limit $\varepsilon \rightarrow 0$, we get

$$
F^{i_{0}}\left(D_{x x}^{2} \varphi_{i_{0}}\left(x_{0}\right), D_{x} \varphi_{i}\left(x_{0}\right), \Lambda^{i} \varphi\left(x_{0}\right), u_{i}\left(x_{0}\right), x_{0}\right) \leq 0 .
$$

\subsection{Viscosity solutions and optimal switching}

The next theorem complements the verification result in Theorem 1.1.

Theorem 2.8. Suppose the value function $\mathbf{u}=\left(u_{1}, u_{2}, \ldots, u_{m}\right)$ in (2) is continuous. Then, $\mathbf{u}$ is a viscosity solution of the system of variational inequalities (3).

Proof. We take a smooth function, $\varphi: \Omega \rightarrow \mathbb{R}^{m}$, and any $\left(x_{0}, i_{0}\right) \in$ $\arg \max _{(x, j) \in \Omega \times\{1, \ldots, m\}}\left(u_{j}(x)-\varphi_{j}(x)\right)$. We want to prove that

$$
\min \left(-L^{i_{0}} \varphi_{i_{0}}\left(x_{0}\right)-f_{i_{0}}\left(x_{0}\right), \min _{j \neq i_{0}}\left(\varphi_{i_{0}}\left(x_{0}\right)-\varphi_{j}\left(x_{0}\right)+\psi_{i_{0} j}\left(x_{0}\right)\right)\right) \leq 0 .
$$

By contradiction, assume that the above inequality does not hold; that is,

$$
-L^{i_{0}} \varphi_{i_{0}}\left(x_{0}\right)-f_{i_{0}}\left(x_{0}\right)>0
$$

and

$$
\varphi_{i_{0}}\left(x_{0}\right)-\varphi_{j}\left(x_{0}\right)+\psi_{i_{0} j}\left(x_{0}\right)>0
$$

for all $j \neq i_{0}$. We consider a ball $B_{\varepsilon}\left(x_{0}\right)$ with radius $\varepsilon$ and center at $x_{0}$, such that $-L^{i_{0}} \varphi_{i_{0}}-f_{i_{0}}>0$ for $x \in B_{\varepsilon}\left(x_{0}\right)$. We can assume that $u_{i_{0}}\left(x_{0}\right)=\varphi_{i_{0}}\left(x_{0}\right)$ and $u_{i}(x)-$ $\varphi_{i}(x) \leq 0$ for all indices $i$ and $x \in \Omega$. From the inequality $\varphi_{i_{0}}\left(x_{0}\right)-\varphi_{j}\left(x_{0}\right)+\psi_{i_{0} j}\left(x_{0}\right)>$ 0 , it follows that $u_{i_{0}}\left(x_{0}\right)-u_{j}\left(x_{0}\right)+\psi_{i_{0} j}\left(x_{0}\right)>0$. By choosing $\varepsilon$ small enough, we can 
also assume that $u_{i_{0}}-u_{j}+\psi_{i_{0} j}>0$ for $x \in B_{\varepsilon}\left(x_{0}\right)$ and all indices $j$. Let $i(\cdot)$ an optimal control for the dynamic programming principle up to the exit time of $B_{\varepsilon}\left(x_{0}\right)$; that is,

$$
u_{i_{0}}\left(x_{0}\right)=E\left[\int_{0}^{T_{\partial B_{\varepsilon}}} f_{i(t)}(x(t)) d t+u_{i\left(T_{\partial B_{\varepsilon}}\right)}\left(x\left(T_{\partial B_{\varepsilon}}\right)\right)\right] .
$$

There is no switching inside the ball because $u_{i_{0}}-u_{j}+\psi_{i_{0} j}>0$. Thus, we apply Dynkin's formula to $\varphi_{i_{0}}$

$$
E \varphi_{i_{0}}\left(x\left(T_{\partial B_{\varepsilon}}\right)\right)-\varphi_{i_{0}}\left(x_{0}\right)=E \int_{0}^{T_{\partial B_{\varepsilon}}} L^{i_{0}} \varphi_{i_{0}}(x(t)) d t<-E \int_{0}^{T_{\partial B_{\varepsilon}}} f_{i_{0}}(x(t)) d t
$$

to get

$$
\varphi_{i_{0}}\left(x_{0}\right)>E \int_{0}^{T_{\partial B_{\varepsilon}}} f_{i_{0}}(x(t)) d t+E u_{i_{0}}\left(x\left(T_{\partial B_{\varepsilon}}\right)\right),
$$

which is a contradiction.

Next, we take a smooth function $\varphi: \Omega \rightarrow \mathbb{R}^{m}$, and any

$$
\left(x_{0}, i_{0}\right) \in \arg \min _{(x, j) \in \Omega \times\{1, \ldots, m\}}\left(u_{j}(x)-\varphi_{j}(x)\right) .
$$

Our aim is to prove that, for all $j \neq i_{0}$, the two following inequalities hold

$$
-L^{i_{0}} \varphi_{i_{0}}\left(x_{0}\right)-f_{i_{0}}\left(x_{0}\right) \geq 0 \text { and } \varphi_{i_{0}}\left(x_{0}\right)-\varphi_{j}\left(x_{0}\right)+\psi_{i_{0} j}\left(x_{0}\right) \geq 0 .
$$

Now, fix $\varepsilon>0$, small enough, and consider the exit time $T_{\partial B_{\varepsilon}}$. From the dynamic programming principle, we have that

$$
u_{i_{0}}\left(x_{0}\right) \geq E\left[\int_{0}^{h \wedge T_{\partial B_{\varepsilon}}} f_{i_{0}}(x(t)) d t+u_{i_{0}}(x(h))\right] .
$$

Accordingly, we have

$$
\varphi_{i_{0}}\left(x_{0}\right)-E \varphi_{i_{0}}(x(h)) \geq E\left[\int_{0}^{h \wedge T_{\partial B_{\varepsilon}}} f_{i_{0}}(x(t)) d t\right] .
$$

After multiplying the preceding inequality by $\frac{1}{h}$ and taking the limit as $h \rightarrow 0$, we get $-L^{i_{0}} \varphi_{i_{0}}\left(x_{0}\right) \geq f_{i_{0}}\left(x_{0}\right)$. To prove the second inequality, we fix a small $h$ and consider the following control:

$$
\begin{cases}i_{h}(t)=i_{0} & \text { for } \quad t \in\left[0, h \wedge T_{\partial B_{\varepsilon}}\right) \\ i_{h}(t)=j & \text { for } \quad t \in\left[h \wedge T_{\partial B_{\varepsilon}}, 2 h \wedge T_{\partial B_{\varepsilon}}\right) .\end{cases}
$$


Then,

$$
\begin{aligned}
& u_{i(t)}\left(x_{0}\right) \geq E\left[\int_{0}^{2 h \wedge T_{\partial B_{\varepsilon}}} f_{i(t)}(x(t)) d t-\psi_{i j}(x(h))+u_{j}\left(x\left(2 h \wedge T_{\partial B_{\varepsilon}}\right)\right)\right] \\
& \geq E\left[\int_{0}^{2 h \wedge T_{\partial B_{\varepsilon}}} f_{i(t)}(x(t)) d t-\psi_{i j}(x(h))+\varphi_{j}\left(x\left(2 h \wedge T_{\partial B_{\varepsilon}}\right)\right)\right] .
\end{aligned}
$$

Thus, by considering the limit $h \rightarrow 0$, we get $\varphi_{i_{0}}\left(x_{0}\right)-\varphi_{j}\left(x_{0}\right)+\psi_{i_{0} j}\left(x_{0}\right) \geq 0$.

\subsection{Penalization method (heuristic)}

The penalized problem corresponding to the optimal switching problem (3) is

$$
-L^{i} u_{i}-\sum \beta_{\varepsilon}\left(-u_{i}^{\varepsilon}+u_{j}^{\varepsilon}-\psi_{i, j}\right)=f_{i}
$$

together with the corresponding Dirichlet boundary conditions. Here, the penalization function $\beta_{\varepsilon}(s)=\beta(s / \varepsilon)$ for all $s \in R, \varepsilon>0$, where $\beta: R \rightarrow[0,+\infty)$ is a smooth convex function such that $\beta(s)=0$ for $s \leq 0$ and $\beta(s)>0$ for $s>0,0<\beta^{\prime}(s) \leq 1$ for $s>0$, and $\lim _{s \rightarrow+\infty} \beta(s)=+\infty$.

To simplify the discussions, we let $m=2$, and $L^{1} \equiv L^{2} \equiv \Delta$. Then, the penalized problem becomes

$$
\left\{\begin{array}{l}
\min \left(-\Delta u_{1}^{\varepsilon}-f_{1}-\beta_{\varepsilon}\left(-u_{1}^{\varepsilon}+u_{2}^{\varepsilon}-\psi_{1}\right)\right)=0 \\
\min \left(-\Delta u_{2}^{\varepsilon}-f_{2}-\beta_{\varepsilon}\left(-u_{2}^{\varepsilon}+u_{1}^{\varepsilon}-\psi_{2}\right)\right)=0
\end{array}\right.
$$

in a given domain and with appropriate boundary data. Moreover, to simplify, we consider the above system with zero boundary data. A similar reasoning is valid for the case with $m$ switching modes using the nonnegative loop conditions and compatibility conditions on the boundary. This is left to the reader to check.

Proposition 2.9. Let $\psi_{1}$ and $\psi_{2}$ satisfy the nonnegative loop condition $\psi_{1}+\psi_{2} \geq 0$ in $\Omega$, and the compatibility condition $\psi_{1} \geq 0$ and $\psi_{2} \geq 0$ on the boundary of $\Omega$. Assume further that $\psi_{1}$ and $\psi_{2}$ have bounded second-order derivatives. Then, the solution of the penalized system (9) with zero Dirichlet boundary data is uniformly bounded.

Proof. Let $\theta_{1}^{\varepsilon}(x)=-u_{1}^{\varepsilon}+u_{2}^{\varepsilon}-\psi_{1}$ and $\theta_{2}^{\varepsilon}(x)=-u_{2}^{\varepsilon}+u_{1}^{\varepsilon}-\psi_{2}$. For $\varepsilon$ fixed, consider the function $\beta_{\varepsilon}\left(\theta_{i}^{\varepsilon}(x)\right), x \in \Omega$. If the maximum of $\beta_{\varepsilon}\left(\theta_{i}^{\varepsilon}(x)\right)$ is attained at the boundary, then

$$
\max _{i=1,2 ; x \in \bar{\Omega}} \beta_{\varepsilon}\left(\theta_{i}^{\varepsilon}(x)\right)=\max _{i=1,2 ; x \in \partial \Omega} \beta_{\varepsilon}\left(-\psi_{i}(x)\right)=0 .
$$

Otherwise, we assume that there exists $x_{0}=x_{0}(\varepsilon) \in \Omega$ such that

$$
\max _{i=1,2 ; x \in \bar{\Omega}} \beta_{\varepsilon}\left(\theta_{i}^{\varepsilon}(x)\right)=\beta_{\varepsilon}\left(\theta_{1}^{\varepsilon}\left(x_{0}\right)\right)>0 .
$$

From the nonnegative loop condition, it follows that $\beta_{\varepsilon}\left(\theta_{2}^{\varepsilon}\left(x_{0}\right)\right)=0$. Since $\beta_{\varepsilon}$ is non- 
decreasing and $\beta_{\varepsilon}(z)>0$ if and only if $z>0$, we have

$$
\max _{x \in \bar{\Omega}} \beta_{\varepsilon}\left(\theta_{i}^{\varepsilon}(x)\right)=\beta_{\varepsilon}\left(\theta_{1}^{\varepsilon}\left(x_{0}\right)\right) .
$$

Equivalently, $-u_{1}^{\varepsilon}+u_{2}^{\varepsilon}-\psi_{1}$ achieves its maximum in $\bar{\Omega}$ at $x_{0}$. Hence, $-\Delta u_{1}+\Delta u_{2}-$ $\Delta \psi_{1} \leq 0$ at $x_{0}$. From the penalized system, we have that

$$
\begin{aligned}
& \beta_{\varepsilon}\left(\theta_{1}^{\varepsilon}\left(x_{0}\right)\right)=-\Delta u_{1}^{\varepsilon}\left(x_{0}\right)-f_{1}\left(x_{0}\right)= \\
& \quad-\Delta u_{1}^{\varepsilon}\left(x_{0}\right)+\Delta u_{2}^{\varepsilon}\left(x_{0}\right)+f_{2}\left(x_{0}\right)-f_{1}\left(x_{0}\right) \leq \Delta \psi_{1}\left(x_{0}\right)+f_{2}\left(x_{0}\right)-f_{1}\left(x_{0}\right) .
\end{aligned}
$$

This proves that $\beta_{\varepsilon}\left(\theta_{i}^{\varepsilon}(x)\right)$ is uniformly bounded. Therefore, $\frac{\theta_{i}^{\varepsilon}(x)}{\varepsilon}$ is uniformly bounded. The above shows that $\Delta u_{i}^{\varepsilon}$ are uniformly bounded. Therefore, by the maximum principle ${ }^{1}$, the functions $u_{i}^{\varepsilon}$ are uniformly bounded. Consequently, $\left\|D u_{i}^{\varepsilon}\right\|_{L^{\infty}(\partial \Omega)}$ are uniformly bounded. From Green's formula it will follow that $\left\|D u_{i}^{\varepsilon}\right\|_{L^{2}(\Omega)}$ are uniformly bounded.

From the proof of the preceding proposition the solution $\left(u_{1}^{\varepsilon}, u_{2}^{\varepsilon}\right)$ of $(9)$ is uniformly bounded and equicontinuous (from the locally uniformly Lipschitz estimates). Thus, by taking the limit through a subsequence as $\varepsilon$ goes to zero, we obtain a continuous viscosity solution, as we state in the theorem below.

Theorem 2.10. The system

$$
\left\{\begin{array}{l}
\min \left(-\Delta u_{1}-f_{1}, u_{1}-u_{2}+\psi_{1}\right)=0 \\
\min \left(-\Delta u_{2}-f_{2}, u_{2}-u_{1}+\psi_{2}\right)=0
\end{array}\right.
$$

has a continuous viscosity solution.

Remark 1. It seems plausible that a similar uniform bound and improved regularity may follow from the following reasoning: define $\theta_{1}^{\varepsilon}:=-u_{1}^{\varepsilon}+u_{2}^{\varepsilon}-\psi_{1}$ and $\theta_{2}^{\varepsilon}:=-u_{2}^{\varepsilon}+$ $u_{1}^{\varepsilon}-\psi_{2}$. Then, under the same conditions as in the above theorem, there should exist a constant $C>0$, such that $\beta_{\varepsilon}\left(\theta_{i}^{\varepsilon}(x)\right) \leq C$ for all $x$ in $\Omega$, all $\varepsilon>0$, and $i=1,2$. We leave the exploration of this to the readers.

\section{A monotone scheme}

Now, we introduce the following iterative procedure to approximate the solutions of (3). First, we set

$$
\left\{\begin{array}{l}
-L u_{1}^{0}-f_{1}=0, \\
\min \left(-L u_{2}^{0}-f_{2}, u_{2}^{0}-u_{1}^{0}+\psi_{21}\right)=0, \\
\min \left(-L u_{3}^{0}-f_{3}, u_{3}^{0}+\min _{j<3}\left(-u_{j}^{0}+\psi_{3 j}\right)\right)=0, \\
\cdots \\
\min \left(-L u_{m}^{0}-f_{m}, u_{m}^{0}+\min _{j<m}\left(-u_{j}^{0}+\psi_{m j}\right)\right)=0,
\end{array}\right.
$$

\footnotetext{
${ }^{1}$ Apply the maximum principle to $u_{i}^{\varepsilon} \pm C v$, for large values of $C$, and $v$ solving $\Delta v=1$, with zero boundary data.
} 
with $\left.u_{i}^{0}\right|_{\partial \Omega}=g_{i}$. Then, inductively, we define

$$
\left\{\begin{array}{l}
\min \left(-L u_{1}^{k}-f_{1}, u_{1}^{k}+\min _{j \geq 1}\left(-u_{j}^{k-1}+\psi_{i j}\right)\right)=0, \\
\cdots \\
\min \left(-L u_{i}^{k}-f_{i}, u_{i}^{k}+\min \left(\min _{j<i}\left(-u_{j}^{k}+\psi_{i j}\right),\right.\right. \\
\left.\left.\min _{j>i}\left(-u_{j}^{k-1}+\psi_{i j}\right)\right)\right)=0, \\
\cdots \\
\min \left(-L u_{m}^{k}-f_{m}, u_{m}^{k}+\min _{j<m}\left(-u_{j}^{k}+\psi_{m j}\right)\right)=0,
\end{array}\right.
$$

with $\left.u_{i}^{k}\right|_{\partial \Omega}=g_{i}$.

Next, we prove that this scheme is convergent and that its limit is a viscosity solution to (3). Our choice of $u_{1}^{0}$ is arbitrary and can be replaced by any function $u_{1}^{0}$ satisfying the boundary conditions and such that $u_{1}^{1} \geq u_{1}^{0}$. As we show later, this property is automatic for our choice of $u_{1}^{0}$. This is crucial since uniqueness in general fails (even for $m=2$ ) if $\psi_{1}+\psi_{2}$ vanishes. Therefore, distinct choices of $u_{1}^{0}$ may give different solutions.

\subsection{Convergence of the approximations}

Now, we show that (14) produces a non-decreasing sequence $\mathbf{u}^{n}$. This fact, coupled with the uniform bounds of Proposition 3.2 below, gives pointwise convergence.

Proposition 3.1. The sequence $u_{i}^{n}$ given by (14) is non-decreasing.

Proof. First, we recall that $u_{1}^{0}$ solves

$$
-L u_{1}^{0}-f_{1}=0
$$

and that $u_{1}^{1}$ solves

$$
\min \left(-L u_{1}^{1}-f_{1}, u_{1}^{1}+\min _{j \geq 1}\left(-u_{j}^{0}+\psi_{i j}\right)\right)=0 .
$$

This implies $u_{1}^{1} \geq u_{1}^{0}$ because the solution of the obstacle problem is larger than the solution without the obstacle. Now, we claim that if $u_{j}^{0} \leq u_{j}^{1}$ for all $j<k$, then $u_{k}^{0} \leq u_{k}^{1}$. For that, we note that $u_{k}^{0}$ solves

$$
\min \left(-L u_{k}^{0}-f_{k}, u_{k}^{0}+\min _{j<k}\left(-u_{j}^{0}+\psi_{k j}\right)\right)=0
$$

and $u_{k}^{1}$ solves

$$
\min \left(-L u_{k}^{1}-f_{k}, u_{k}^{1}+\min \left(\min _{j<k}\left(-u_{j}^{1}+\psi_{i j}\right), \min _{j>k}\left(-u_{j}^{0}+\psi_{i j}\right)\right)\right)=0 .
$$

Consequently, $u_{k}^{0}$ has obstacle $\min _{j<k}\left(-u_{j}^{0}+\psi_{k j}\right)$, whereas $u_{k}^{1}$ has obstacle

$$
\min \left(\min _{j<k}\left(-u_{j}^{1}+\psi_{i j}\right), \min _{j>k}\left(-u_{j}^{0}+\psi_{k j}\right)\right) .
$$


Because

$$
-u_{j}^{0}+\psi_{k j} \geq-u_{j}^{1}+\psi_{k j}
$$

and

$$
\min _{j<k}\left(-u_{j}^{0}+\psi_{k j}\right) \geq \min _{j<k}\left(-u_{j}^{1}+\psi_{k j}\right)
$$

we conclude that $u_{k}^{0} \leq u_{k}^{1}$. The preceding inductive argument is valid for arbitrary $j$. Therefore, we have $u_{k}^{l} \leq u_{k}^{l+1}$.

To establish uniform bounds for the sequence $u^{n}$ given by (14), we require that either the obstacle functions are positive, that is,

$$
\psi_{i j}(x) \geq 0
$$

or they are smooth enough

$$
\psi_{i j} \in C^{2}
$$

As stated in the introduction, in this last case, we work under the no-profit loop condition, (5).

Proposition 3.2. If (15) or (16) and (5) hold then the sequence $u^{n}$ given by (14) is uniformly bounded by above.

Proof. We consider first the case $\psi_{i j}>0$. In this case, we show that $u_{i}^{k} \leq M:=$ $\max \left\{\sup g_{j}, u_{j}^{0}\right\}$ for all $k=0,1,2, \ldots$ and $i=1, \ldots, m$. This statement is obvious for $k=0$. Thus, we proceed by induction. For that, we fix $1 \leq i \leq m$ and assume that $u_{r}^{k-1} \leq M$, for $1 \leq r \leq m$ and $u_{j}^{k} \leq M$ for $1 \leq j<i$. Recall that $u_{i}^{k}$ is the solution of the following obstacle problem:

$$
\left\{\begin{array}{l}
\min \left(-L u_{i}^{k}-f_{i}, u_{i}^{k}+\min \left(\min _{j<i}\left(-u_{j}^{k}+\psi_{i j}\right), \min _{j>i}\left(-u_{j}^{k-1}+\psi_{i j}\right)\right)\right)=0, \\
u_{i}^{k}=g_{i} .
\end{array}\right.
$$

Let $A_{k}$ be the coincidence set of the preceding obstacle problem:

$$
A_{k}=\left\{x \in \Omega: u_{i}^{k}+\min \left(\min _{j<i}\left(-u_{j}^{k}+\psi_{i j}\right), \min _{j>i}\left(-u_{j}^{k-1}+\psi_{i j}\right)\right)=0\right\} .
$$

Because $u_{i}^{k}$ solves the obstacle problem, we have $-L u_{i}^{k}-f_{i} \geq 0$ in the noncoincidence set. Thus, if the maximum of $u_{i}^{k}$ is achieved at $z \in \bar{\Omega}$, by the maximum principle either $z$ belongs to the coincidence set or to the boundary of the noncoincidence set. The boundary points of the non-coincidence set are the points either from $\partial \Omega$ or that belong to the $A_{k}$ the coincidence set. In both these cases, $u_{k}(x) \leq M$. Therefore,

$$
u_{i}^{k}=\max \left(\max _{j<i}\left(u_{j}^{k}-\psi_{i j}\right), \min _{j>i}\left(u_{j}^{k-1}-\psi_{i j}\right)\right) \leq M
$$


Now, we consider the general case when the functions $\psi_{i j}$ can change the sign. For any $x_{0} \in \Omega$, there exists a sequence $j_{i}$ such that

$$
-u_{j_{i}}^{k\left(j_{i}\right)}+\psi_{i j_{i}}=\min \left(\min _{j<i}\left(-u_{j}^{k}+\psi_{i j}\right), \min _{j>i}\left(-u_{j}^{k-1}+\psi_{i j}\right)\right),
$$

where $k\left(j_{i}\right)=k$ if $j_{i}<i$ or $k\left(j_{i}\right)=k-1$ if $j_{i}>i$. We may assume that $j_{i}=i+1$, this is possible to do as in the other case we will re-enumerate the system. Then, (14) becomes

$$
\left\{\begin{array}{l}
\min \left(-L u_{1}^{k}-f_{1}, u_{1}^{k}-u_{j_{1}}^{k\left(j_{1}\right)}+\psi_{1 j_{1}}\right)=0, \\
\cdots \\
\min \left(-L u_{i}^{k}-f_{i}, u_{i}^{k}-u_{j_{i}}^{k\left(j_{i}\right)}+\psi_{i j_{i}}\right)=0, \\
\cdots \\
\min \left(-L u_{m}^{k}-f_{m}, u_{m}^{k_{m}}-u_{j_{m}}^{k\left(j_{m}\right)}+\psi_{m j_{m}}\right)=0 .
\end{array}\right.
$$

Let $h$ be a $C^{2}$ function satisfying

$$
-\operatorname{Lh}(x)=1, \quad h(x) \geq 1, \quad \psi(x) \leq C h(x)
$$

for some $C$ large such that $f(x)-L \psi(x)+C \geq 1$; here, we assume $\psi \in C^{2}$. Now, we set $v_{1}^{k}=u_{1}^{k}+\psi_{1 j_{1}}+h+M$ and $v_{j_{1}}^{k\left(j_{1}\right)}=u_{2}^{n}+h+M$. Next, we define $u_{i}^{k}-u_{j_{i}}^{k\left(j_{i}\right)}+\psi_{i j_{i}}=$ $v_{i}^{k}-v_{j_{i}}^{k\left(j_{i}\right)}$ and $u_{m}^{k_{m}}-u_{j_{m}}^{k\left(j_{m}\right)}+\psi_{m j_{m}}=v_{i}^{k}-v_{j_{i}}^{k\left(j_{i}\right)}+\sum_{i} \psi_{i j_{i}}$.

Because of the no-profit loop condition, the new variables, $v_{1}^{k}$, convert the system into a new one with positive obstacles. Therefore, the preceding part of the proof applies.

Theorem 3.3. If (15) or (16) and (5) hold then the limit function

$$
\mathbf{u}=\left(u_{1}, \ldots, u_{m}\right)=\lim _{n \rightarrow \infty} u^{n}(x)
$$

is a viscosity solution of (3). If we additionally require strict positivity loop condition then the function $\mathbf{u}$ continuous.

Proof. Recall that a function $\mathbf{u}$ is lower semicontinuous if

$$
\mathbf{u}(x) \leq \mathbf{u}_{*}(x):=\liminf _{y \rightarrow x} \mathbf{u}(y)
$$

The limit of a bounded increasing sequence of continuous functions is lower semicontinuous and coincide with its lower semicontinuous envelop $\mathbf{u}(x)=\mathbf{u}_{*}(x)$. Now, we show that for $\mathbf{u}$ as in the statement, $\mathbf{u}^{*}$ is a sub-solution to (3). For that, we argue by contradiction and suppose $\mathbf{u}^{*}$ is not a sub-solution. Accordingly, there exists a degree two polynomial $\varphi$ and a point $\left(x_{0}, i_{0}\right) \in \arg \min _{(x, i) \in \Omega \times\{1 \ldots m\}}\left(u_{i}(x)-\varphi_{i}(x)\right)$ (for simplicity let set $i_{0}=1$ ) such that

$$
\min \left\{-L \varphi_{1}\left(x_{0}\right)-f_{1}\left(x_{0}\right), \varphi_{1}\left(x_{0}\right)+\min _{j}\left(-\varphi_{j}\left(x_{0}\right)+\psi_{1 j}\left(x_{0}\right)\right)\right\}>0 .
$$


Then,

$$
-L \varphi_{1}\left(x_{0}\right)-f_{1}\left(x_{0}\right)>0
$$

and

$$
u_{1}^{*}\left(x_{0}\right)>u_{j}^{*}\left(x_{0}\right)-\psi_{1 j}\left(x_{0}\right), \quad j=1 \ldots m .
$$

Because $u^{k} \uparrow \mathbf{u}^{*}$, for $\mu$ small enough and $k$ large enough, we have

$$
u_{1}^{k}\left(x_{0}\right)-u_{j}^{k-1}\left(x_{0}\right)>-\psi_{1 j}\left(x_{0}\right)+\mu .
$$

Because $f, \psi$, and $u_{j}^{k}$ are continuous, there exists $r>0$ such that

$$
\begin{gathered}
-L \varphi_{1}(x)-f_{1}(x)>0, \quad x \in B\left(x_{0}, r\right), \\
u_{1}^{k}(x)-u_{j}^{k-1}(x)>-\psi_{1 j}(x)+\mu, \quad x \in B\left(x_{0}, r\right) .
\end{gathered}
$$

For a smooth function $\eta(x)$ with the property $L \eta=1, \eta \geq 0$ and $\eta\left(x_{0}\right)=0$, we set

$$
\varphi_{1, \varepsilon}=\varphi_{1}+\varepsilon \eta(x) .
$$

If $\varepsilon>0$ is small enough, then

$$
-L \varphi_{1, \varepsilon}(x)-f_{1}(x)>0, \quad x \in B\left(x_{0}, r\right) .
$$

Let us show that,

$$
\varphi_{1, \varepsilon}(x)>\varphi_{1}(x) \geq u_{1}^{*}(x), \quad x \neq x_{0} .
$$

Using the fact that $u_{k} \uparrow \mathbf{u}^{*}$, we can choose $k$ large enough such that

$$
\inf _{B\left(x_{0}, r\right)}\left(\varphi_{1, \varepsilon}-u_{k}\right)<\inf _{\partial B\left(x_{0}, r\right)}\left(\varphi_{1, \varepsilon}-\mathbf{u}\right)
$$

Moreover, as $\mathbf{u}^{*}\left(x_{0}\right)=\varphi_{1, \varepsilon}\left(x_{0}\right)$ and $\mathbf{u}^{*}$ is an upper semi-continuous function, we have

$$
\inf _{B\left(x_{0}, r\right)}\left(\varphi_{1, \varepsilon}-u_{k}\right)<\mu .
$$

Now, we define $Q_{\varepsilon}=\varphi_{1, \varepsilon}-c$, where $c \in \mathbb{R}$ is chosen such that $Q_{\varepsilon}$ touches $u_{k}$ from above at a point $x^{\prime} \in B\left(x_{0}, r\right)$. By (20), the first touching point in $B\left(x_{0}, r\right)$ cannot be on the boundary of $B\left(x_{0}, r\right)$.

We observe that $Q_{\varepsilon}$ satisfies the following conditions:

$$
\begin{gathered}
Q_{\varepsilon}(x) \geq u_{k}(x), \quad x \in B\left(x_{0}, r\right), \\
Q_{\varepsilon}\left(x^{\prime}\right)=u_{k}\left(x^{\prime}\right), \quad \text { where } x^{\prime} \in B\left(x_{0}, r\right) .
\end{gathered}
$$


Because $u_{k}$ is a viscosity subsolution of

$$
\min \left\{-L u_{1}^{k}-f_{1}, u_{1}^{k}(x)+\min _{j>1}\left(-u_{j}^{k-1}(x)+\psi_{1 j}(x)\right)\right\}=0,\left.\quad u_{1}^{k}\right|_{\partial \Omega}=g_{1},
$$

the definition of viscosity subsolution and (22)-(23) give

$$
\min \left\{-L Q_{\varepsilon}\left(x^{\prime}\right)-f_{1}\left(x^{\prime}\right), Q_{\varepsilon}\left(x^{\prime}\right)+\min _{j>1}\left(-u_{j}^{k-1}(x)+\psi_{1 j}(x)\right)\right\} \leq 0 .
$$

From (19) it follows that $-L Q_{\varepsilon}\left(x^{\prime}\right)-f_{1}\left(x^{\prime}\right)>0$. Consequently, the only possibility to have (24) is that

$$
Q_{\varepsilon}\left(x^{\prime}\right)+\min _{j>1}\left(-u_{j}^{k-1}\left(x^{\prime}\right)+\psi_{1 j}\left(x^{\prime}\right)\right) \leq 0 .
$$

There exist $j_{0}$ such that

$$
\varphi_{1}\left(x^{\prime}\right) \leq u_{j_{0}}^{k-1}\left(x^{\prime}\right)-\psi_{1 j_{0}}\left(x^{\prime}\right)+c-\varepsilon \eta(x) .
$$

Then

$$
u_{1}^{k}\left(x^{\prime}\right) \leq u^{*}\left(x^{\prime}\right) \leq \varphi_{1}\left(x^{\prime}\right) \leq u_{j_{0}}^{k-1}\left(x^{\prime}\right)-\psi_{1 j_{0}}\left(x^{\prime}\right)+c-\varepsilon \eta(x)
$$

and

$$
u_{1}^{k}\left(x^{\prime}\right) \leq u_{j_{0}}^{k-1}\left(x^{\prime}\right)-\psi_{1 j_{0}}\left(x^{\prime}\right)+c .
$$

However, from (18), we have that

$$
u_{1}^{k}\left(x^{\prime}\right)>u_{2}^{k-1}\left(x^{\prime}\right)-\psi_{1}\left(x^{\prime}\right)+\mu .
$$

By equation (21), we have $c<\mu$, which is a contradiction, and it follows that $\mathbf{u}^{*}$ is a sub-solution of (3).

Now, we show that $u=\mathbf{u}_{*}$ is a supersolution, see (17). Accordingly, we argue by contradiction and assume that $u$ is not a super-solution. Then, as before, there exists a degree-two polynomial $\varphi$ and a point

$$
\left(x_{0}, i_{0}\right) \in \underset{(x, i) \in \Omega \times\{1,2\}}{\arg \min }\left(u_{i}(x)-\varphi_{i}(x)\right)
$$

(for simplicity, set $i_{0}=1$ ) such that

$$
\min \left\{-L \varphi_{1}\left(x_{0}\right)-f_{1}\left(x_{0}\right), u_{1}\left(x_{0}\right)+\min _{j}\left(-u_{j}\left(x_{0}\right)+\psi_{1 j}\left(x_{0}\right)\right)\right\}<0 .
$$

Then,

$$
-L \varphi_{1}\left(x_{0}\right)-f_{1}\left(x_{0}\right)<0
$$




$$
u_{1}\left(x_{0}\right)+\min _{j}\left(-u_{j}\left(x_{0}\right)+\psi_{1 j}\left(x_{0}\right)\right)<0 .
$$

We consider first the former inequality. Because $\varphi_{1}$ and $f$ are continuous, there exists $r>0$ such that

$$
-L \varphi_{1}(x)-f_{1}(x)<0, \quad x \in B\left(x_{0}, r\right) .
$$

As previously, we construct a polynomial $Q=\varphi_{1}-c$ that touches $u_{k}$ at $x^{\prime} \in B\left(x_{0}, r\right)$; that is,

$$
\begin{gathered}
Q(x) \leq u_{k}(x), \quad x \in B\left(x_{0}, r\right) \\
Q\left(x^{\prime}\right)=u_{k}\left(x^{\prime}\right), \quad \text { where } \quad x^{\prime} \in B\left(x_{0}, r\right) .
\end{gathered}
$$

The function $u_{1}^{k}$ is a viscosity supersolution of

$$
\min \left\{-L u_{1}^{k}-f_{1}, u_{1}^{k}(x)+\min _{j>1}\left(-u_{j}^{k-1}(x)+\psi_{1 j}(x)\right)\right\}=0
$$

which led us to a contradiction as $-L Q-f_{1}<0$.

Finally, we show that the inequality (25) also cannot hold. For that, let $j_{0}$ be an integer where the minimum of the second term in (25) is achieved. Consequenty,

$$
u_{1}\left(x_{0}\right)-u_{j_{0}}\left(x_{0}\right)<\psi_{1 j_{0}}\left(x_{0}\right) .
$$

As $u_{k} \uparrow \mathbf{u}^{*}$, we can choose $k$ large enough such that

$$
u_{1}^{k}\left(x_{0}\right)-u_{j_{0}}^{k-1}\left(x_{0}\right)<\psi_{1 j_{0}}\left(x_{0}\right) .
$$

The function $u_{1}^{k}(x)-u_{j_{0}}^{k-1}(x)$ is continuous in $\Omega$. Thus, there exists $N$ such that if $k>N$ then

$$
u_{1}^{k}(x)-u_{j_{0}}^{k-1}(x)<\psi_{1 j_{0}}(x), \quad x \in B\left(x_{0}, r\right) .
$$

The function $u_{1}^{k}(x)$ satisfies (26) which is impossible due to previous inequality.

The comparison principle (see [7] Theorem 3.1) implies that if we have strict positivity loop condition then super-solution should be greater or equal to the sub-solution. From the definition of $\mathbf{u}$ it follow that $\mathbf{u}^{*} \geq \mathbf{u}$, so $\mathbf{u}=\mathbf{u}_{*}$ is a continuous viscosity solution of (3).

\section{Existence and Uniqueness examples}

To the authors' best knowledge, paper [1] was the first to consider the existence problem for weakly coupled systems. However, in that paper, only the case of positive (or vanishing) switching costs is addressed. The general case with non-negative switching 
costs was addressed in $[2,3]$. Furthermore, in $[3,6]$, the authors obtained a uniqueness result for the case where there are no loops of zero switching cost.

In our first example, we show that, even when the transition costs are non-negative, a solution to (3) may fail to exist.

Example 4.1 (Lack of existence). Consider the following one-dimensional weakly coupled system in $\Omega=[-1,1]$ :

$$
\left\{\begin{array}{l}
\min \left(-\left(u_{1}\right)_{x x}, u_{1}-u_{2}+|| x|-1| \cos \left(2 \pi \frac{1}{1-|x|}\right)\right)=0 \\
\min \left(-\left(u_{2}\right)_{x x}, u_{2}-u_{1}-|| x|-1| \cos \left(2 \pi \frac{1}{1-|x|}\right)\right)=0
\end{array}\right.
$$

By contradiction, we assume that there exists a solution, $u$, to the preceding system. Because $u$ is a viscosity solution to (27), we have

$$
u_{i}(x) \leq E \sum_{0 \leq t \leq T_{\partial \Omega}} \psi_{i\left(t^{-}\right), i\left(t^{+}\right)}(x(t))
$$

where $x(t)$ is a Brownian motion started at $x, T_{\partial \Omega}$ is the first hitting time to $\partial \Omega$, and $i(t) \in\{1,2\}$ is any process with a countable number of discontinuities.

Because $T_{\partial \Omega}<\infty$ with probability one, there are infinite number of instances $t_{k}$ for which

$$
\cos \left(2 \pi \frac{1}{1-\left|x\left(t_{k}\right)\right|}\right)= \pm 1
$$

Therefore, at these instances, the transition cost is ||$x\left(t_{k}\right)|-1|= \pm \frac{1}{n}$. Then, by maximizing, we get $u=+\infty$ because $\sum \frac{1}{n}$, the total transition cost, would diverge. Consequently, no solution can exist.

Next, we examine two cases where the uniqueness does not hold. In both cases, there are loops with zero switching costs.

Example 4.2 (Degenerate case). Consider the problem

$$
\left\{\begin{array}{l}
\min \left(-\Delta u_{1}-M, u_{1}-u_{2}+\psi\right)=0 \\
\min \left(-\Delta u_{2}+M, u_{2}-u_{1}-\psi\right)=0
\end{array}\right.
$$

Assume zero boundary data for $u_{1}$ and $u_{2}$ and that $\psi$ vanishes in the boundary. Suppose $u_{1}(x)$ vanishes on the boundary and is such that

$$
\Delta u_{1}+M<0 .
$$

Then, $u_{2}$ is given by

$$
u_{2}(x)=u_{1}(x)+\psi(x) .
$$

If $M$ is large, we also have

$$
-\Delta u_{2}+M>0
$$


Thus, uniqueness of solution does not hold.

Example 4.3 (Vanishing at one point). Let $\Omega=[-1,1]$, and $u_{i}(-1)=u_{i}(1)=0$, solving

$$
\min \left(-\left(u_{1}\right)_{x x}, u_{1}-u_{2}+|x|\right)=0, \quad \min \left(-\left(u_{2}\right)_{x x}, u_{2}-u_{1}+|x|\right)=0 .
$$

Then

$$
u_{i}(x)=a(1-|x|)
$$

with $a \geq 0$ is a viscosity solution.

Obviously, $-\Delta u_{i} \geq 0$ in viscosity sense, and vanishes for all $x \neq 0$. We also have

$$
u_{1}-u_{2}+|x| \geq 0
$$

everywhere and vanishing at $x=0$.

\section{Financial Bubbles}

Now, we examine optimal switching problems that arise in the study of speculative financial bubbles, proposed in [26]. In this model, a trader profits from the overvaluation of certain stocks by other traders and, thus, trading agents "agree to disagree" on the value of assets. Moreover, agents are overconfident in the sense that they believe that their valuations have a higher degree of confidence. Finally, there are no restrictions on short selling. As a result, asset prices can exceed their fundamental values.

In [26], the authors consider the one-dimensional stationary case and construct an explicit solution using Kummer functions. Related stationary models were considered in $[27,28]$. Subsequently, a multidimensional stationary version of the model was introduced in [29] where an iterative scheme was suggested for its numerical solution. Here, we consider the problem discussed in [29] in a more general framework and reformulate it in terms of a switching problem. We consider the system

$$
\begin{cases}\min (-L u(x)+f(x), u(x)-u(-x)-\psi(x))=0, & x \in \Omega \\ u(x)=g(x), & x \in \partial \Omega\end{cases}
$$

where $L$ is an elliptic operator of the form

$$
L u=a^{i j}(x) D_{i j} u+b^{i}(x) D_{i} u+c(x) u,
$$

with $a^{i, j}=a^{j, i}$, the matrix $\left[a^{i, j}(x)\right]$ is positive definite for all $x \in \Omega$ and the coefficients $a^{i, j}, b^{i}, c$ are continuous. Furthermore, we assume that the operator is symmetric; that is,

$$
\begin{aligned}
& (L \widetilde{u})(x)=a^{i j}(x) D_{i j} u(-x)-b^{i}(x) D_{i} u(-x)+c(x) u(-x) \equiv \\
& \quad a^{i j}(-x) D_{i j} u(-x)+b^{i}(-x) D_{i} u(-x)+c(-x) u(-x)=(L u)(-x)=(\widetilde{L u})(x),
\end{aligned}
$$

where $\widetilde{u}(x)=u(-x)$. 
Finally, we assume the following compatibility condition at the boundary:

$$
g(x)-g(-x)+\psi(x) \geq 0
$$

on $\partial \Omega$.

\subsection{A model problem}

To illustrate the connection between the asset bubble and optimal switching problems, we let

$$
\begin{aligned}
\psi_{1}(x) \equiv \psi(x), & \psi_{2}(x) \equiv \psi(-x), \\
g_{1}(x) \equiv g(x), & g_{2}(x) \equiv g(-x), \\
f_{1}(x) \equiv f(x), & f_{2}(x) \equiv f(-x)
\end{aligned}
$$

be smooth functions. Then, (30) can be written as

$$
\left\{\begin{array}{l}
\min \left(-L u_{1}+f_{1}, u_{1}-u_{2}+\psi_{1}\right)=0 \\
\min \left(-L u_{2}+f_{2}, u_{2}-u_{1}+\psi_{2}\right)=0
\end{array}\right.
$$

with the boundary conditions $\left.u^{i}\right|_{\partial \Omega}=g^{i}$, where $L$ is defined in (31), and has the symmetry property (32). We assume further $\psi_{1}+\psi_{2} \geq 0$ in $\Omega$. A simpler situation occurs when there is $\gamma>0$ such that $\psi_{1}+\psi_{2} \geq \gamma$ in $\Omega$. In this last case, (33) has a unique solution (see Section 4).

Theorem 5.1. If there are no loops of zero switching costs, i.e. $\psi_{1}+\psi_{2} \geq 0$, then problems (30) and (33) are equivalent.

Proof. As both (30) and (33) have a unique solution, it is enough to show that if $u$ solves (30) then $v=\left(v_{1}, v_{2}\right)=(u(x), u(-x))$ solves (33). First, observe that $u$ is a viscosity subsolution. Hence, for any pair $\left(x_{0}, \varphi_{1}\right)\left(\varphi_{1} \in C^{2}(\Omega)\right.$ and $\left.x_{0} \in \Omega\right)$ such that $u\left(x_{0}\right)=\varphi_{1}\left(x_{0}\right)$ and

$$
u \leq \varphi_{1} \text { on } \Omega,
$$

we have

$$
\min \left(-L \varphi_{1}\left(x_{0}\right)+f\left(x_{0}\right), u\left(x_{0}\right)-u\left(-x_{0}\right)-\psi\left(x_{0}\right)\right) \leq 0 .
$$

Moreover, the same inequality holds for the pair $\left(-x_{0}, \varphi_{2}\right)$; that is,

$$
\min \left(-L \varphi_{2}\left(-x_{0}\right)+f\left(-x_{0}\right), u\left(-x_{0}\right)-u\left(x_{0}\right)-\psi\left(-x_{0}\right)\right) \leq 0 .
$$


Combining above inequalities, we have

$$
\left\{\begin{array}{l}
\min \left(L \varphi_{1}, \varphi_{1}\left(x_{0}\right)-\varphi_{2}\left(x_{0}\right)+a\left(x_{0}\right)\right) \leq 0, \\
\min \left(L \varphi_{2}, \varphi_{2}\left(x_{0}\right)-\varphi_{1}\left(x_{0}\right)+a\left(-x_{0}\right)\right) \leq 0 .
\end{array}\right.
$$

Consequently, $\left(v_{1}, v_{2}\right)$ is a viscosity subsolution of $(33)$; i.e., for any smooth function $\varphi$ and for any

$$
\left(x_{0}, i_{0}\right) \in \underset{(x, i) \in \Omega \times\{1, \ldots, m\}}{\arg \min }\left(u_{i}(x)-\varphi_{i}(x)\right),
$$

we have

$$
\min \left(L \varphi_{i_{0}}, \varphi_{i_{0}}\left(x_{0}\right)-\varphi_{i_{1}}\left(x_{0}\right)+a\left(x_{0}\right)\right) \leq 0 .
$$

In a similar way, we can prove the supersolution property, which concludes the proof.

\section{Numerical Results}

In this last section, we present numerical computations that illustrate the behavior of the iterative scheme. Each step of our scheme involves solving an obstacle problem using a finite-difference method.

To construct a finite-difference scheme, we discretize $\Omega$ using a regular uniform mesh. In one dimension, we approximate the Laplace operator as follows: for any interior point, $i$, we set

$$
L_{h} u_{i}=\frac{u_{i-1}-2 u_{i}+u_{i+1}}{h^{2}} .
$$

In the two-dimensional case, we use a 5-point stencil for the Laplacian:

$$
L_{h} u_{i, j}=\frac{u_{i-1, j}+u_{i+1, j}-4 u_{i, j}+u_{i, j-1}+u_{i, j+1}}{h^{2}} .
$$

Using these discretizations in (14), we obtain the following nonlinear system:

$$
\left\{\begin{array}{l}
\min \left(-L_{h} u_{\alpha}^{k+1}+f_{\alpha}, u_{\alpha}^{k+1}-u_{\alpha}^{k}-\psi_{\alpha}\right)=0 \\
u_{k+1}^{\alpha}=g^{\alpha}
\end{array}\right.
$$

where $f_{\alpha}, \psi_{\alpha}$, and $g_{\alpha}$ are the values at the nodes of $f(x), \psi(x)$, and $g(x)$, and $u_{\alpha}^{k}$ is the finite-difference approximation of (14). 
Example 6.1. Let $\Omega=[0,1]$, we consider the following problem:

$$
\left\{\begin{array}{l}
\min \left\{-u_{1}^{\prime \prime}(x)-60 \sin (12 x), \min _{j \neq 1}\left(u^{1}-u^{j}+1 / 2\right)\right\}=0 \\
\min \left\{-u_{2}^{\prime \prime}(x)-50 \cos (9 x), \min _{j \neq 2}\left(u^{2}-u^{j}+1 / 2\right)\right\}=0 \\
\min \left\{-u_{3}^{\prime \prime}(x)-45 \sin (10 x), \min _{j \neq 3}\left(u^{3}-u^{j}+1 / 2\right)\right\}=0 \\
u_{1}(0)=0.3, u_{1}(1)=0.1, \\
u_{2}(0)=0, u_{2}(1)=0.5 \\
u_{3}(0)=0.4, u_{2}(1)=0.2
\end{array}\right.
$$

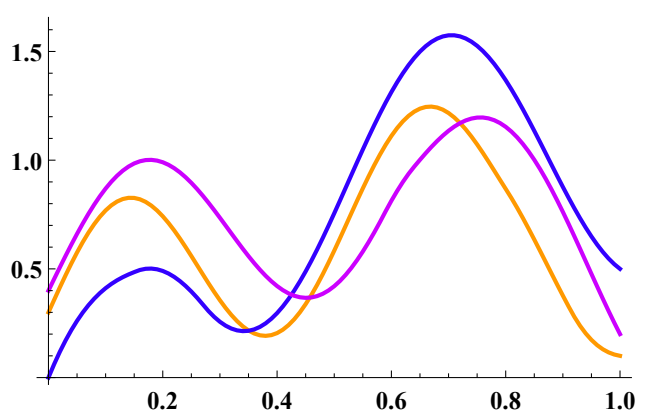

(a) Numerical solution of the example 6.1

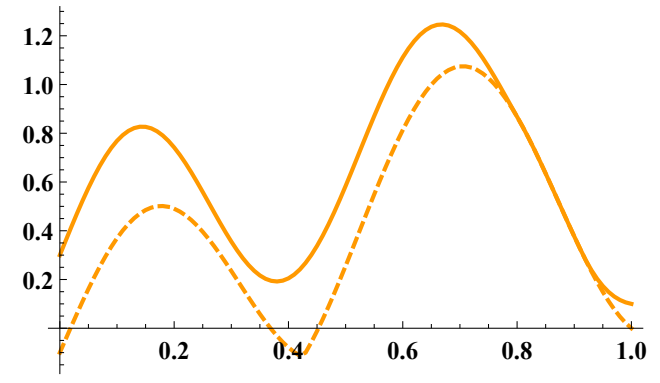

(b) $u_{1}$ and the obstacle function $\max _{j \neq 1}\left(u^{j}-1 / 2\right)$

Figure 1. Numerical results for Example 6.1.

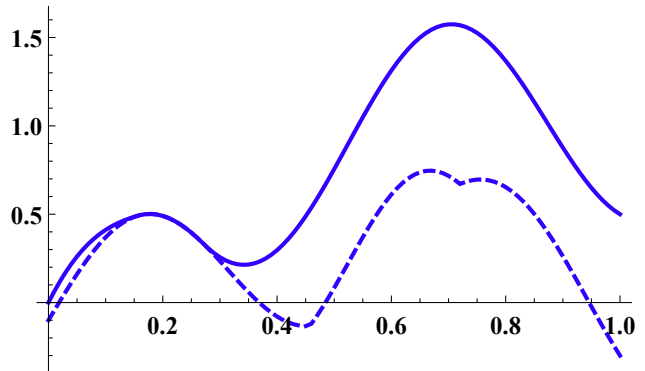

(a) $u_{2}$ and the obstacle function $\max _{j \neq 2}\left(u^{j}-1 / 2\right)$

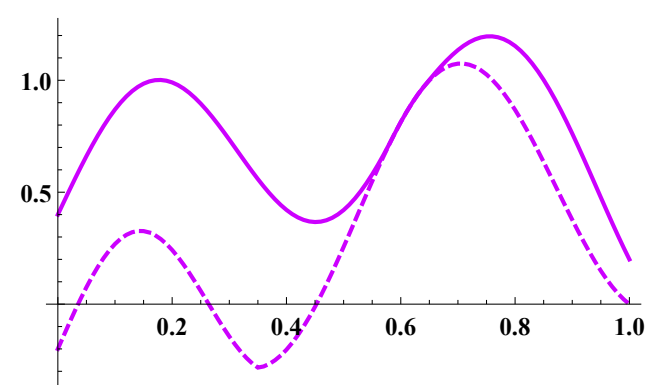

(b) $u_{3}$ and the obstacle function $\max _{j \neq 3}\left(u^{j}-1 / 2\right)$

Figure 2. Numerical results for Example 6.1.

The iterative algorithm gives the solution for (35) represented in Figure 1a. The graph of the functions $u_{1}, u_{2}, u_{3}$ are shown, respectively, in solid orange, purple and blue lines. The calculation corresponds to 5 steps of iteration and the finite-difference scheme is applied with $N=100$ points. In Figure $1 \mathrm{~b}$, the function $u_{1}$ and the obstacle function for the first equation is shown in dashed orange line; that is, $\max _{j \neq 1}\left(u^{j}-1 / 2\right)$ are presented. In Figures $2 \mathrm{a}$ and $2 \mathrm{~b}$, we depict the functions $u_{2}, u_{3}$ and the obstacle function $\max _{j \neq 2}\left(u^{j}-1 / 2\right), \max _{j \neq 3}\left(u^{j}-1 / 2\right)$.

The functions $u_{1}, u_{2}$ and $u_{3}$ consist of two parts: the first part, where they touch the dashed obstacle (the coincidence sets) $\max _{j \neq 1}\left(u^{j}-1 / 2\right), \max _{j \neq 2}\left(u^{j}-1 / 2\right)$ or $\max _{j \neq 3}\left(u^{j}-1 / 2\right)$, 
respectively, and the second part, where they are above the obstacles and hence the solutions to the differential equations (non-coincidence sets). The points, that divide the coincidence set from non-coincidence set form the free boundary of the problem.

Next, we consider one-dimensional financial bubble problem and illustrate the connections between these two problems. Let $\Omega=[-1,1]$.

\section{Example 6.2.}

$$
\left\{\begin{array}{l}
\min \left(-u^{\prime \prime}(x)+\sin (x), u(x)-u(-x)-\psi(x)\right)=0 \\
u(-1)=1.01, \quad u(1)=1.11
\end{array}\right.
$$

where $\psi(x)=0.1 x-0.05$.

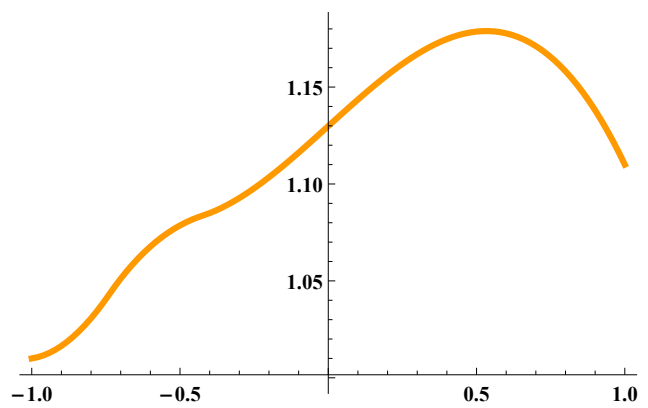

(a) Numerical solution of Example 6.2

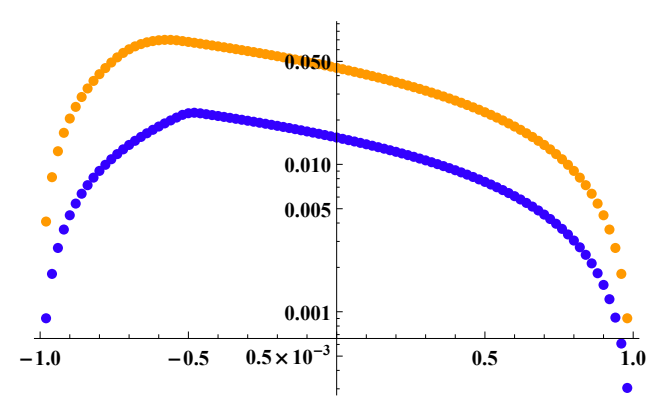

(b) Differences between the second and the third step of the iteration

Figure 3. Numerical results for Example 6.2.

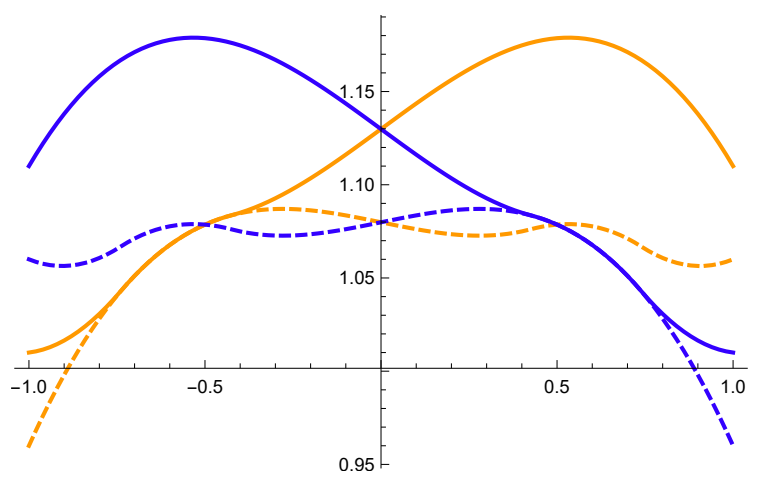

Figure 4. Switching problem corresponding to Example 6.2

In Figure 3a, we show the numerical solution of Example 6.2. The results correspond to 5 steps of iteration and the finite difference scheme is applied with $N=100$ points. In Figure 3b , we show the difference between second and third steps of iteration.

In Figure 4, we present the solution of the optimal switching problem in Example 6.2; the function $u_{1}=u$ corresponds to the solid orange line and the corresponding obstacle is the dashed orange line $u_{1}(-x)+\psi(x)$, the function $u_{2}=u(-x)$ is the blue line with the obstacle $u_{1}(x)+\psi(-x)$.

Lastly, we consider a two-dimensional problem. 


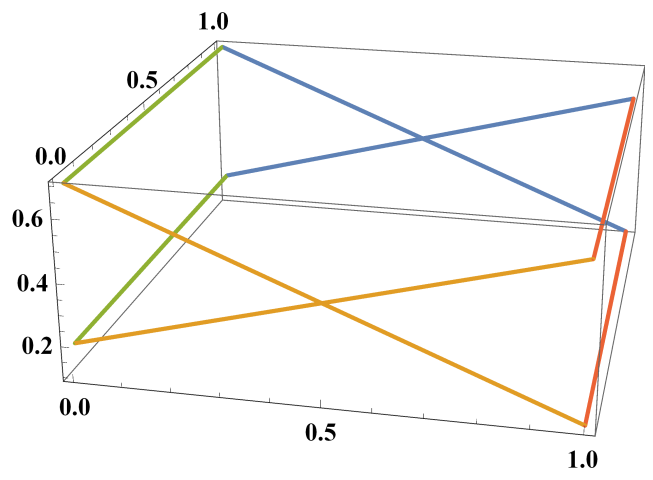

(a) Boundary values

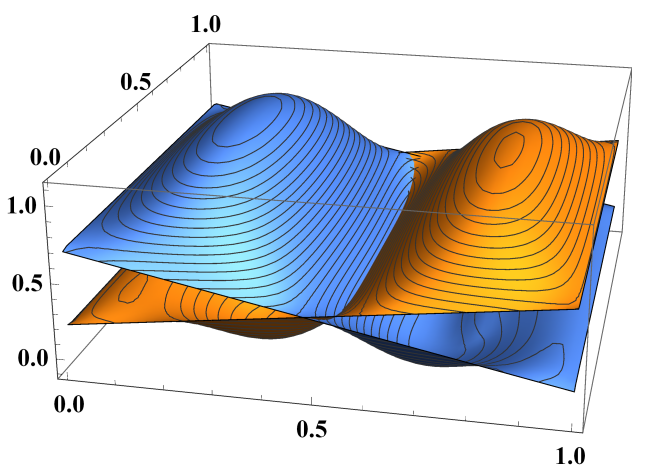

(b) Numerical solution

Figure 5. Boundary values and the numerical solution of Example 6.3

Example 6.3. Let $\Omega=[0,1] \times[0,1]$ and consider

$$
\left\{\begin{array}{l}
\min \left\{-\Delta u_{1}(x)-30 \cos (8 x), u^{1}-u^{2}+1\right\}=0 \\
\min \left\{-\Delta u_{2}(x)+30 \cos (8 x+1), u^{2}-u^{1}+1\right\}=0 \\
u_{i}=g_{i} \text { on the boundary }
\end{array}\right.
$$

where

$$
g_{1}(x, 0)=.2, g_{1}(x, 1)=.6, g_{2}(0, x)=g_{2}(1, x)=0.2+0.4 x
$$

and

$$
g_{2}(x, 0)=.7, g_{2}(x, 1)=0.1, g_{2}(0, x)=g_{2}(1, x)=0.7-0.6 x \text {. }
$$

In Figures 5a, 5b, we depict the boundary data and the numerical solution. Figure

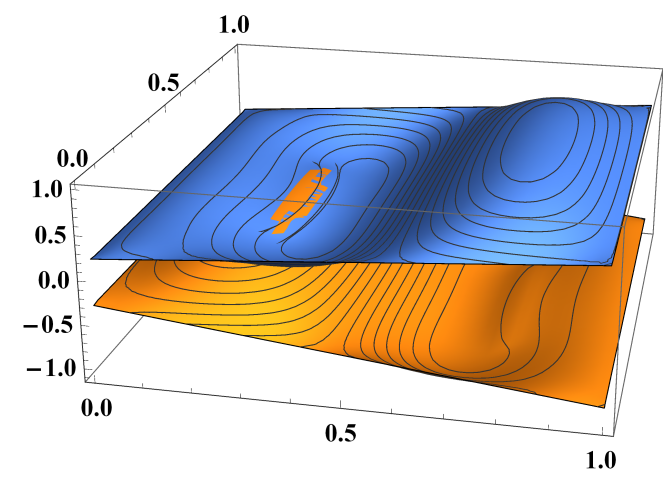

(a) Numerical solution with the obstacle

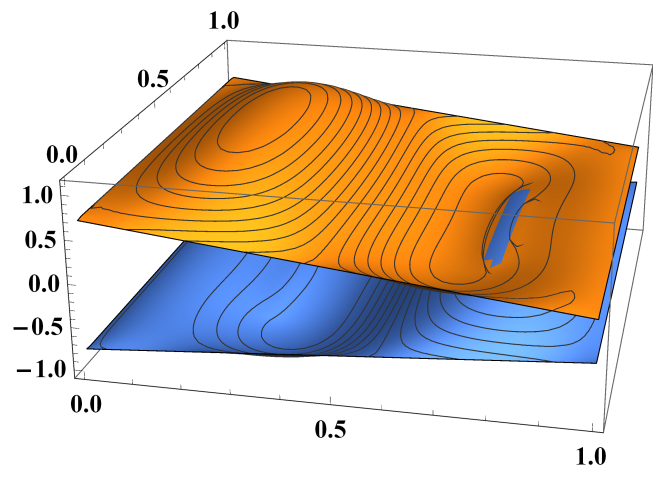

(b) Numerical solution with the obstacle

Figure 6. Numerical solutions and obstacle functions of Example 6.3

$5 \mathrm{~b}$ was constructed with a $50 \times 50$ discretization and 5 iterations.

Figures $6 \mathrm{a}$ and $6 \mathrm{~b}$ show the corresponding level sets of $u_{1}(x), u_{2}(x)-1$ and $u_{2}(x)$, $u_{1}(x)-1$. 


\section{Acknowledgement}

This work was partially supported by Qatar National Research Fund (a member of Qatar Foundation) [grant number NPRP 5-088-1-021]. The statements made herein are solely the responsibility of the authors.

\section{References}

[1] Evans LC, Friedman A. Optimal stochastic switching and the Dirichlet problem for the Bellman equation. Trans Amer Math Soc. 1979;253:365-389.

[2] Belbas SA, Lenhart SM. Nonlinear PDEs for stochastic optimal control with switchings and impulses. Appl Math Optim. 1986;14(3):215-227. Available from: http://dx.doi.org/10.1007/BF01442237.

[3] Lenhart SM, Belbas SA. A system of nonlinear partial differential equations arising in the optimal control of stochastic systems with switching costs. SIAM J Appl Math. 1983; 43(3):465-475. Available from: http://dx.doi.org/10.1137/0143030.

[4] Yamada N. A system of parabolic variational inequalities associated with a stochastic switching game. Nonlinear Anal. 1985;9(1):39-51. Available from: http://dx.doi.org/10.1016/0362-546X(85)90051-3.

[5] Yamada N. A system of elliptic variational inequalities associated with a stochastic switching game. Hiroshima Math J. 1983;13(1):109-132. Available from: http://projecteuclid.org/getRecord?id=euclid.hmj/1206133541.

[6] Ishii H, Koike S. Viscosity solutions for monotone systems of second-order elliptic PDEs. Comm Partial Differential Equations. 1991;16(6-7):1095-1128.

[7] Ishii H, Koike S. Viscosity solutions of a system of nonlinear second-order elliptic PDEs arising in switching games. Funkcial Ekvac. 1991;34(1):143-155. Available from: http://www.math.kobe-u.ac.jp/ fe/xml/mr1116886.xml.

[8] Liu Wa, Chen H. Viscosity solutions of nonlinear systems of degenerated elliptic equations of second order. Z Anal Anwendungen. 2000;19(4):927-951.

[9] Weian L, Gang L, Hua C, et al. Viscosity solutions of fully nonlinear degenerated elliptic systems. Commun Appl Anal. 2003;7(2-3):299-312.

[10] Liu W, Yang Y, Lu G. Viscosity solutions of fully nonlinear parabolic systems. J Math Anal Appl. 2003;281(1):362-381.

[11] Yamada N. Viscosity solutions for a system of elliptic inequalities with bilateral obstacles. Funkcial Ekvac. 1987;30(2-3):417-425. Available from: http://www.math.kobeu.ac.jp/ fe/xml/mr0927191.xml.

[12] Camilli F, Loreti P, Yamada N. Systems of convex Hamilton-Jacobi equations with implicit obstacles and the obstacle problem. Commun Pure Appl Anal. 2009;8(4):1291-1302. Available from: http://dx.doi.org/10.3934/cpaa.2009.8.1291.

[13] Arnarson T, Djehiche B, Poghosyan M, et al. A PDE approach to regularity of solutions to finite horizon optimal switching problems. Nonlinear Anal. 2009;71(12):6054-6067. Available from: http://dx.doi.org/10.1016/j.na.2009.05.063.

[14] Djehiche B, Hamadène S, Popier A. A finite horizon optimal multiple switching problem. SIAM Journal on Control and Optimization. 2009;48(4):2751-2770. Available from: http://dx.doi.org/10.1137/070697641.

[15] Djehiche B, Hamadène S. On a finite horizon starting and stopping problem with risk of abandonment. Int J Theor Appl Finance. 2009;12(4):523-543. Available from: http://dx.doi.org/10.1142/S0219024909005312.

[16] Barles G, Evans LC, Souganidis PE. Wavefront propagation for reaction-diffusion systems of PDE. Duke Math J. 1990;61(3):835-858.

[17] Quaas A, Sirakov B. Solvability of monotone systems of fully nonlinear elliptic PDE's. C R Math Acad Sci Paris. 2008;346(11-12):641-644. 
[18] Busca J, Sirakov B. Harnack type estimates for nonlinear elliptic systems and applications. Ann Inst H Poincaré Anal Non Linéaire. 2004;21(5):543-590.

[19] de Figueiredo DG, Sirakov B. Liouville type theorems, monotonicity results and a priori bounds for positive solutions of elliptic systems. Math Ann. 2005;333(2):231-260.

[20] El Asri B, Fakhouri I. Optimal multi-modes switching with the switching cost not necessarily positive. arXiv:12041683v1 [mathOC]. 2012;.

[21] El Asri B, Fakhouri I. Viscosity solutions for a system of PDEs and optimal switching. IMA J Math Control Inform. 2017;34(3):937-960. Available from: https://doi.org/10.1093/imamci/dnw004.

[22] Lundström NLP, Olofsson M, Önskog T. Existence, uniqueness and regularity of solutions to systems of nonlocal obstacle problems related to optimal switching. J Math Anal Appl. 2019;475(1):13-31. Available from: https://doi.org/10.1016/j.jmaa.2018.11.003.

[23] Lundström NLP, Nyström K, Olofsson M. Systems of variational inequalities for non-local operators related to optimal switching problems: existence and uniqueness. Manuscripta Math. 2014;145(3-4):407-432. Available from: https://doi.org/10.1007/s00229-014-06839.

[24] Lundström NLP, Nyström K, Olofsson M. Systems of variational inequalities in the context of optimal switching problems and operators of Kolmogorov type. Ann Mat Pura Appl (4). 2014;193(4):1213-1247. Available from: https://doi.org/10.1007/s10231-013$0325-\mathrm{y}$.

[25] Martyr R. Finite-horizon optimal multiple switching with signed switching costs. Math Oper Res. 2016;41(4):1432-1447. Available from: https://doi.org/10.1287/moor.2016.0783.

[26] Scheinkman JA, Xiong W. Overconfidence and speculative bubbles. Journal of Political Economy. 2003;111(6):pp. 1183-1220. Available from: http://www.jstor.org/stable/10.1086/378531.

[27] Chen X, Kohn RV. Erratum to: Asset price bubbles from heterogeneous beliefs about mean reversion rates [mr2800215]. Finance Stoch. 2013;17(1):225-226. Available from: http://dx.doi.org/10.1007/s00780-012-0191-2.

[28] Chen X, Kohn RV. Asset price bubbles from heterogeneous beliefs about mean reversion rates. Finance Stoch. 2011;15(2):221-241. Available from: http://dx.doi.org/10.1007/s00780-010-0124-x.

[29] Barkhudaryan R, Juráš M, Salehi M. Iterative scheme for an elliptic non-local free boundary problem. Applicable Analysis. 2016;95(12):2794-2806. Available from: http://dx.doi.org/10.1080/00036811.2015.1113528. 